\title{
Relationship between Wheatstone Bridge Circuit and Femtogram Particles Attached on Piezoresistive Microcantilever in Biosensor Application
}

\author{
Ratno Nuryadi* \\ Center of Material Technology, Agency for the Assessment and Application of Technology, 2nd BPPT Building 22 Floor, M.H. Thamrin 8 Street, Jakarta 10340, Indonesia \\ Received 29 July 2011, Revised 5 October 2011, Accepted 11 November 2011, Available online 30 November 2011
}

\begin{abstract}
This paper describes basic calculations that are used in a piezoresistive microcantilever for the biosensor application. The working principle of the sensor is generally based on piezoresistance change due to the change of stress in the microcantilever. Such stress change is caused by the microcantilever deflection induced by the adsorption of the ultrasmall particles (virus, glucose, micro-organism, etc) on the microcantilever surface. In this work, two piezoresistors embedded in the microcantilever and two external resistors are used to build the Wheatstone bridge circuit for detecting the stress change. Basic equations in stress analysis and in Wheatstone bridge are considered in order to explain the relationship between the Wheatstone bridge and the particles attached on microcantilever surface. Calculation results show that the particles with the mass of femtogram results in piezoresistance change in the order of $\mu \Omega$ and the voltage output in the order of $\mu \mathrm{V}$.
\end{abstract}

| piezoresistive microcantilever | biosensor | Wheatstone bridge | femtogram particles | stress change

(B) 2011 Ibnu Sina Institute. All rights reserved. http://dx.doi.org/10.11113/mjfas.v7n2.245

\section{INTRODUCTION}

Recent semiconductor technology enables us to fabricate micro- and nano-sized cantilevers, which can be used as biosensor in the medicine, chemistry and biology fields [1-3]. The advantage of this detection method over the other diagnosis system is not only ultrasensitive but also less time consuming and more energy efficient process. It has been reported that the sensitivity of the microcantilever is enough to detect a single virus particle $[4,5]$. Moreover, functionalizing of microcantilever arrays with different analyte receptors can also allow for the detection of multiple various objects on the same chip. Basic operation of the microcantilever-based sensor is the detection of the cantilever deflection [6] or resonance frequency shift [4] due to the attachment of the particles (virus, glucose, microorganism, etc.) on the microcantilever surface. There are several types for measuring the cantilever deflection or resonance frequency shift such as optical detection, piezoresistive, capacitance and piezoelectric methods. Recently, piezoresistive microcantilever has progressively reported because it can overcome the difficulties in laser alignment of the optical detection, so that the small size biosensor with simple detection system can be realized. In the piezoresistive method, the change of piezoresistance due to the microcantilever deflection is usually detected by the Wheatstone bridge circuit [7-9].

Corresponding author at: Center of Material Technology

Agency for the Assessment and Application of Technology, Jakarta, Indonesia

E-mail addresses: ratnon@gmail.com (Ratno Nuryadi)
In order to understand the working mechanism of piezoresistive microcantilever, the material properties including the piezoresistance gauge factor dan crystal composition, and the Wheatstone bridge circuit need to be analyzed. The purpose of this work is to investigate the relationship between the Wheatstone bridge circuit and the picogram particles attached on the surface of the piezoresistive microcantilever. First, a basic principle of the piezoresistive microcantilever is introduced. Next, the electronic circuit that is used in the piezoresistive microcantilever for detecting the objects (Wheatstone bridge circuit) is described. Finally, example calculation for microcantilever geometry of $110 \mu \mathrm{m} \times 40 \mu \mathrm{m} \times 1 \mu \mathrm{m}$ and experimental results from the Wheatstone bridge circuit are discussed.

\section{PIEZORESISTIVE MICROCANTILEVER}

Piezoresistive microcantilever-based biosensor measures the piezoresistance change occured in the microcantilever due to the deflection-induced strain. The change in the piezoresistance $\Delta R$ is proportional to the strain occured in the microcantilever, as shown in the equation below [10],

$\Delta R=G s R$,

where $G$ is the piezoresistance gauge factor, $R$ is the resistance of the piezoresistor and $s$ is the strain. 
The piezoresistance gauge factor $G$ is determined by multiplying the piezoresistance with Young's Modulus in the direction of the applied strain. The single-crystal silicon and polycrystalline silicon are usually used in the fabrication of microcantilever. The piezoresistance coefficients of single-crystalline silicon are not constant, but are influenced by the doping concentration, type of dopant and temperature [11-13]. So far, the values of piezoresistive coefficient matrices $\left(\pi_{11}, \pi_{12}\right.$ and $\left.\pi_{44}\right)$ for single-crystalline silicon under certain doping concentration and dopant types have been already reported [14].

In this work, piezoresistive microcantilever with property of p-type single crystal silicon (resistivity 7.8 $\Omega \mathrm{cm},<110>$ direction) is considered. In case of a longitudinal piezoresistor embedded on the top surface of microcantilever as shown in Fig. 1, the longitudinal piezoresistance coefficient can be calculated as follows [14],

$$
\begin{aligned}
\pi_{l, 110} & =\frac{1}{2}\left(\pi_{11}+\pi_{12}+\pi_{44}\right) \\
& =\frac{1}{2}(6.6-1.1+138.1) \times 10^{-11} \\
& =71.8 \times 10^{-11} \mathbf{P a}^{-1} .
\end{aligned}
$$

Since the Young's modulus of single-crystalline silicon in the $<110>$ direction is $168 \mathrm{GPa}$, the effective gauge factor $G$ is

$$
G=71.8 \times 10^{-11}\left(\mathrm{~Pa}^{-1}\right) \times 168 \times 10^{9}(\mathrm{~Pa})=120.6 .
$$

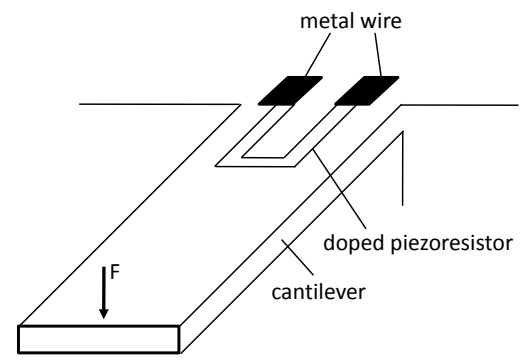

Fig. 1 Schematic view of piezoresistive microcantilever made from single crystal silicon.

Next, a force $F$ is applied in the free-end of the microcantilever beam. The magnitude of the maximum stress is expressed as

$\sigma_{\max }=\frac{M t}{2 I}$

Here, $M$ is torque, $t$ is microcantilever thickness and $I$ is moment of inertia. The torque $M$, moment of inertia $I$ and the maximum strain $s_{\max }$ for microcantilever in Fig. 1 can be then calculated as follows:

$\boldsymbol{M}=\boldsymbol{F l}$

$I=\frac{w t^{3}}{12}$

$s_{\max }=\frac{\sigma_{\max }}{E}$
Therefore, equation (1) becomes

$\frac{\Delta R}{R}=\frac{G F l t}{2 E I}$

If the particle with mass of $m$ gram attached on the free-end cantilever surface, the force will be $F=N m g$, where $N$ is number of particle and $g$ is gravitation force. In this case, the equation (4) can then be written as:

$\frac{\Delta R}{R}=\frac{G N m g l t}{2 E I}$

Using equation (5), the change of piezoresistance due to number of particles $N$ attached on the free-end of microcantilever is evaluated. The microcantilever with size of $110 \mu \mathrm{m} \times 40 \mu \mathrm{m} \times 1 \mu \mathrm{m}$ is used. It is noted that the size of microcantilever is the same as the microcantilever made by Seiko Instrument. The cantilever is made from single crystal silicon (resistivity $7.8 \Omega \mathrm{cm},<110>$ direction). It is also assumed that the value of piezoresistance is $630 \Omega$. Figure 2 shows the calculated results of the change in piezoresistance $\Delta R$ and the strain $s$ as a function of $N$. It can be seen that the particles with the mass of femtogram level cause the $\Delta R$ and stress $s$ in the order of $\mu \Omega$ and several tens $\mathrm{N} / \mathrm{m}^{2}$, respectively. Both $\Delta R$ and stress $\sigma$ increase with increasing $N$, indicating that large number of particles results in larger sensor output.

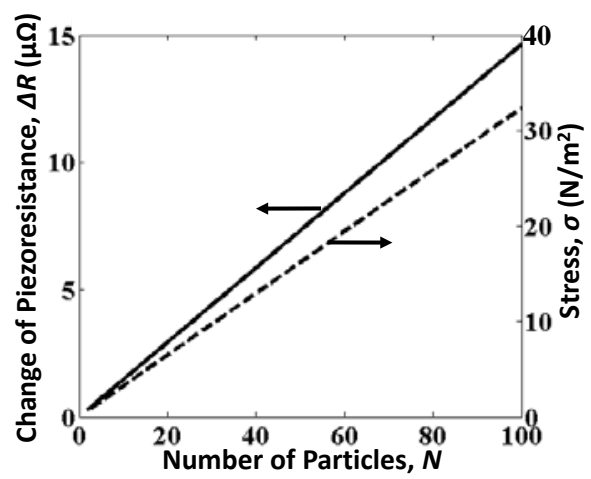

Fig. 2 Calculated results of change in piezoresistance $\Delta R$ and stress $\sigma$ as a function of number of particles $N$ attached on the free-end of the microcantilever.

\section{WHEATSTONE BRIDGE}

After the change of piezoresistance is known, it is important to evaluate the output voltage from the microcantilever. The change of piezoresistance in the microcantilever is usually measured by the Wheatstone bridge circuit. Commercial cantilever such as Seiko Instrument has two piezoresistances embedded in the cantilever. One is designed for the deflection detection, while the other one is designed as a reference. It is noted that the piezoresistance of the reference does not change when the microcantilever deflects. 
The Wheatstone bridge circuit is constructed using two piezoresistors $\left(R_{1}\right.$ and $\left.R_{2}\right)$ in the microcantilever and two external resistors $\left(R_{3}\right.$ and $\left.R_{4}\right)$, as shown in Fig. 3. When the bridge is fully balanced, which is obtained for $R_{1}=R_{3}$ and $R_{2}=R_{4}$, the voltage across the bridge will be zero. This is called balance condition $(\Delta V=0)$. However, a small change in resistance on the one of the piezoresistors results in the unbalanced bridge, and a voltage difference $\Delta V$ appears.

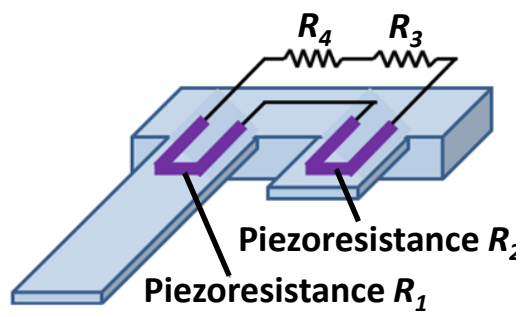

(a)

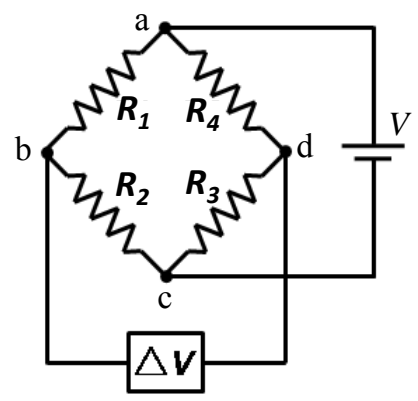

(b)

Fig. 3 (a) Illustration of piezoresistive microcantilever with two external resistors and (b) Wheatstone bridge circuit constructed from two piezoresistors embedded in microcantilever and two external resistors.

Next, the detail equations in the Wheatstone bridge is presented. For a given voltage input $V$, the currents flowing through $a b c$ and $a d c$ (see Fig. 3(b)) depend on the resistances, i.e.,

$V=V_{a b c}=V_{a d c}$

From equation above, it is obtained that,

$V=I_{a b c}\left(R_{1}+R_{2}\right)=I_{a d c}\left(R_{3}+R_{4}\right)$

The voltage drops from $a$ to $b$ and from $a$ to $d$ are given by,

$V_{a b}=I_{a b c} R_{1}=\frac{R_{1}}{\left(R_{1}+R_{2}\right)} V$
$V_{a d}=I_{a d c} R_{x}=\frac{R_{x}}{\left(R_{3}+R_{4}\right)} V$

The voltage difference $\Delta V$ can then be obtained from,

$\Delta V=V_{a b}-V_{a d}$
$\Delta V=\frac{R_{1}}{\left(R_{1}+R_{2}\right)} V-\frac{R_{4}}{\left(R_{3}+R_{4}\right)} V$
$\Delta V=\frac{R_{1} R_{3}-R_{2} R_{4}}{\left(R_{1}+R_{2}\right)\left(R_{3}+R_{4}\right)} V$

Assuming that $R_{1}=R_{3}=R+\Delta R$ and $R_{2}=R_{4}=R$, the voltage difference $\Delta V$ is given by [4-6],

$\Delta V=\frac{2 R \Delta R+\Delta R^{2}}{(2 R+\Delta R)^{2}} V$

If the value of $R$ is much larger than $\Delta R, \Delta V$ becomes,

$$
\Delta V=\frac{\Delta R}{2 R} V
$$

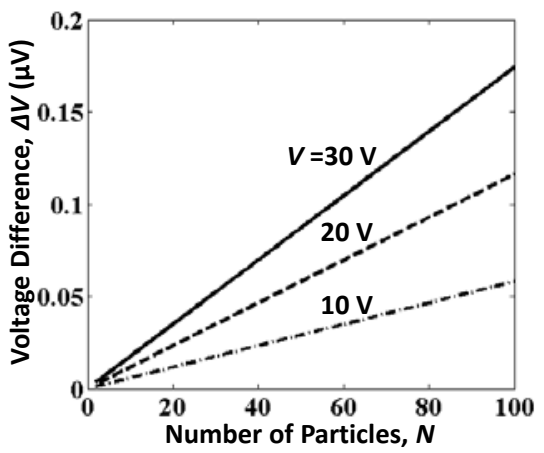

Fig. 4 Calculated results of voltage difference $\Delta V$ from the Wheatstone bridge as a function of $N$ particles for three different voltage inputs.

Equation (13) gives the relationship between $\Delta V$ and $\Delta R$. The output data of $\Delta V$ can be obtained when the value of $\Delta R$ is known. The change in the piezoresistance $\Delta R$ can be calculated from equation (5), so that equation (13) becomes,

$\Delta V=\frac{G N m g l t}{4 E I} V$

Figure 4 shows the calculated results of the voltage difference $\Delta V$ as a function of $N$ for three voltage inputs of $10 \mathrm{~V}, 20 \mathrm{~V}$ and $30 \mathrm{~V}$. Problem used in this calculation is the same with that of the calculation in Fig. 2. It can be seen that voltage output of $\mu \mathrm{V}$ level is found when femtogram particles attached on the free-end of the microcantilever. As number of particles $N$ increases, the voltage difference increases. It is also seen that the large voltage input results in large voltage difference. It is noted that the piezoresistive microcantilever with the size of $110 \mu \mathrm{m} \times 40 \mu \mathrm{m} \times 1 \mu \mathrm{m}$ from Seiko Instrument enables us to measure the femtogram mass particles [8].

Next, the experiment for testing the Wheatstone bridge circuit built by the piezoresistive microcantilever is presented. Here, measurement of the microcantilever deflection is done by using potentiometer which replaces a function of piezoresistance in the microcantilever. The circuit is shown in Fig. 5. Resistors $R_{1}$ and $R_{2}$ are assumed to be piezoresistors within microcantilever, while $R_{3}, R_{4}$ and $R_{5}$ are an external resistors. The output signal of the Wheatstone bridge is then multiplied by the operational 
amplifier. The power supply and digital multimeters are used in order to operate and to measure the Wheatstone bridge circuit.

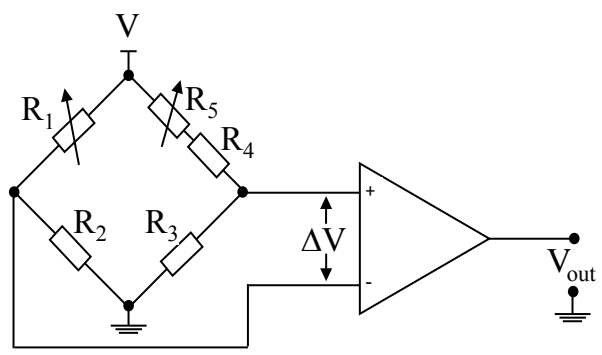

Fig. 5 Wheatstone bridge circuit constructed from two piezoresistors in the microcantilever and two external resistors. A small value of $\Delta V$ is then amplified by the operational amplifier.

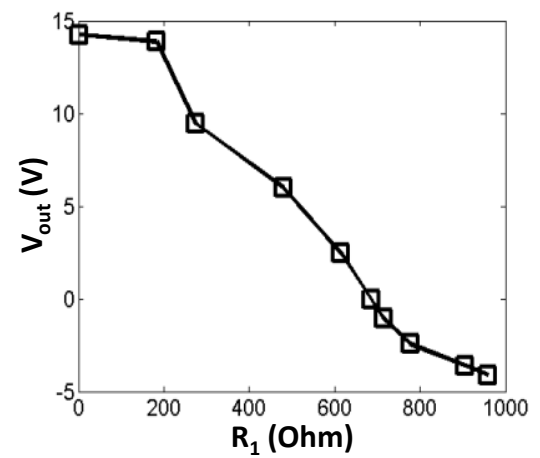

Fig. 6 Experimental result of the Wheatstone bridge output voltage $V_{\text {out }}$ as a function of $R_{1}$ in Fig. 5.

In Fig. 5, a value of $\Delta V$ is obtained and then it is delivered into the operational amplifier. The output voltage
$\mathrm{V}_{\text {out }}$ is measured and then the value of $\Delta V$ can be calculated from the gain operated in the operational amplifier.

In the experiment, first, the value of $R_{1}$ is set to be about $630 \Omega$ and then $\mathrm{V}_{\text {out }}$ is set to be zero by adjusting the potentiometer $R_{5}$ to obtain the equilibrium condition. Next, the value of $V_{\text {out }}$ is measured by the changing the values of $R_{1}$. Figure 6 shows the relationships between $V_{\text {out }}$ and $R_{1}$ curve. Here, voltage input $V$ is set to be $-6.9 \mathrm{~V}$. It can be seen that both $V_{\text {out }}$ and $\Delta V$ decrease with increasing $R_{1}$. Because the gain of operational amplifier is 5 times, the valus of $\Delta V$ will be 5 times smaller than that of $V_{\text {out. }}$ It is also found very small noise, so that the Wheatstone bridge circuit built here can be used for microcantilever biosensor application.

\section{CONCLUSION}

In this work, the basic analysis of the piezoresistive microcantilever for biosensor application was done. Especially, the electronic circuit detector based on the Wheatstone bridge using piezoresistive microcantilever was evaluated. In this circuit, two piezoresistors embedded in the microcantilever and two external resistors are used. The relationship between the output of the Wheatstone bridge and femtogram particles attached on the microcantilever surface was studied. It was found that femtogram particles produce the piezoresistance change in the order of $\mu \Omega$ and the output of the Wheatstone bridge in the order of $\mu \mathrm{V}$. Finally, the experimental results from the Wheatstone bridge was also discussed.

\section{ACKNOWLEDGEMENT}

I would like to thank our colleagues, Mr. Himma Firdaus and Mr. Aan Ferdiansyah for their contributions as well as to Indonesia State Ministry of Research and Technology for funding the project.

\section{REFERENCES}

[1] N.R. Frómeta, Bio-tecnología Aplicada, 23 (2006) 320-323.

[2] N.V. Larvik, M.J. Sepaniak, P.G. Datskos, Rev. Sci. Instrum, 75 (2004) 2229-2250.

[3] C. Wang, D. Wang, Y. Mao, X. Hu, Anal Biochem, 363 (2007) 1-11.

[4] A. Gupta, D. Akin, R. Basir, Appl. Phys. Lett., 84-11 (2004) 1976-1978.

[5] B. Ilic, HG. Craighead, S. Krylov, W. Senaratne, C. Ober, P. Neuzil, J. of Appl. Phys., 95 (2004) 3694-3703.

[6] J. Fritz, M.K. Baller, H.P. Lang, H. Rothuizen, P. Vettiger, E. Meyer, H.J. Guntherodt, C. Gerber, J.K. Gimzewski, Science, 288 (5464) (2000) $316-318$.

[7] M. Tortonese, R.C. Barrett, C.F. Quate, Appl. Phys. Lett., Vol. 62 (1993) 834-836.

[8] S. Hosaka, T. Chiyoma, A. Ikeuchi, H. Okano, H. Sone, T. Izumi, Current Appl. Phys., 6 (2006) 384-388.

[9] H. Sone, A. Ikeuchi, T. Izumi, H. Haruki, S. Hosaka, Japanese J. Appl. Phys., 45 No 3B (2006) 2301-2304.

[10] Chang Liu, Foundation of MEMS, Prentice Hall Publisher, 2006.

[11] Y. Kanda, Sensors and Actuators A: Physical, 28 (1991) 83-91.

[12] K. Yamada, M. Nishihara, S. Shimada, IEEE Trans. Electron Devices, ED-29 (1982) 71-77.

[13] O.N. Tufte, E.L. Stelzer, J. of Appl. Phys., 34 (1963) 313-318.

[14] S.D. Senturia, Microsystem Design, Kluwer Academic Publisher, 2001. 